\title{
Sistemas Descentralizados Integrados y Sostenibles para el Tratamiento de Aguas Residuales Domesticas
}

\section{Sustainable Integrated Systems Decentralized for the Treatment of Residual Waters Domiciliary}

\author{
Eduardo Valencia G ${ }^{1}$ Ivonne Julieth Silva G. ${ }^{2}$ y Clara Patricia \\ Narváez R. ${ }^{3}$
}

\begin{abstract}
Resumen
En el sector rural del departamento del Huila, las aguas residuales de las diferentes actividades generalmente son vertidas crudas a las fuentes hídricas causando problemas de contaminación y salud pública. Tradicionalmente se ha propuesto alcantarillados y sistemas de tratamiento convencionales y centralizados, caracterizados por ser de alto costo. Como otra alternativa de solución, este estudio propone los Sistemas Descentralizados Integrados y Sostenibles, que se caracterizan por integrar el Tratamiento con el Reuso y la Producción, además de ser de bajo costo y fácil construcción, operación y mantenimiento. Se presentan tres prototipos de Sistemas Descentralizados Integrados y Sostenibles para el tratamiento de aguas residuales domésticas, que contemplan como tratamiento secundario Humedales, Albercas Biológicas y Tanques Sépticos de Acción Múltiple, con eficiencias teóricas de remoción superiores a $80 \%$ en DBO, SS, G y A y $50 \%$ en N y P; convirtiéndose en una alternativa para la descontaminación de las microcuencas.
\end{abstract}

Palabras clave: Aguas Residuales; Tratamiento; Sistemas Integrados.

\begin{abstract}
In the rural sector of the department of Huila, the wastewater of different activities are usually discharged raw water sources causing pollution problems and public health. Traditionally have been proposed sewage treatment systems and conventional, centralized, characterized by its high cost. As another alternative solution, this study proposes the Integrated and Sustainable Decentralized Systems, characterized by integrating the Reclamation Treatment and Production, in addition to being low cost and easy construction, operation and maintenance. There are three prototypes of integrated and sustainable decentralized systems for treating domestic wastewater, which provide secondary treatment as Wetlands, Biological Pools and Multiple Action Septic Tanks, with theoretical efficiencies greater than $80 \%$ removal of BOD, $\mathrm{SS}, \mathrm{G}$ and $\mathrm{A}$ and $50 \%$ in $\mathrm{N}$ and $\mathrm{P}$; becoming an alternative for the decontamination of the watersheds.
\end{abstract}

Keywords: Wastewater; Treatment; Integrated Systems.

1 Magíster en Ingeniería Sanitaria y Ambiental. Docente Universidad Surcolombiana Neiva. Avenida Pastrana Carrera $1^{a}$ eduvale@usco.edu.co 2 Ingeniera Agrícola.Universidad Surcolombiana Neiva. tjivonne@hotmail.com

3 Ingeniera Agrícola. Universidad Surcolombiana Neiva.. jaclar1122@hotmail.com

Revista Ingeniería y Región No. 7 Año 2010 


\section{Introducción}

En Colombia, generalmente los pequeños municipios y el sector rural presentan problemas de contaminación del recurso hídrico, como consecuencia de la baja inversión en proyectos de saneamiento básico. Los sistemas de tratamiento de aguas residuales que se han diseñado y construido tanto en el sector urbano como rural se caracterizan por ser centralizados, de alto costo y exigente operación y mantenimiento.

En el sector rural, la cobertura en alcantarillado y tratamiento de aguas residuales es baja. Comparativamente es más costoso construir alcantarillados en el sector rural que en el sector urbano; y construir sistemas centralizados y convencionales para el tratamiento de aguas residuales, además de los costos se dificulta aún más por la operación y mantenimiento. Aunque se construyen alcantarillados y sistemas de tratamiento centralizados en la zona rural, no siempre es posible por razones de tipo topográfico y económico. En el sector rural también se construyen sistemas de tratamiento de aguas residuales descentralizados, siendo el caso más común los tanques sépticos.

En las zonas de minifundio del departamento del Huila, se generan aguas residuales que son vertidos a las fuentes hídricas, sin ningún tipo de tratamiento, dando como resultado problemas de salud y de contaminación. Como una alternativa a la problemática mencionada, en los últimos años, se han propuesto diferentes tipos de sistemas que tienen de común ser no convencionales y descentralizados. En el presente proyecto se proponen los sistemas descentralizados integrados y sostenibles para el tratamiento de aguas residuales del sector rural en el departamento del Huila, y tres prototipos de los mismos que contemplan como sistemas secundarios Humedales, Alberca Biológica y Tanque Séptico de Acción Múltiple.

Los Sistemas Integrados Sostenibles para el Tratamiento de Aguas residuales son procesos en los cuales se integra TRATAMIENTO - REUSO - PRODUCCION, y los subproductos del tratamiento son nuevamente utilizados por el hombre, minimizando así la contaminación. (Valencia y Olaya, 1998).Los sistemas integrados de tratamiento de aguas residuales se definen como una combinación de procesos y prácticas donde el uso óptimo del recurso se alcanza vía el reciclaje de desechos, el cual, se logra mediante la recuperación y reuso de los nutrientes y la energía. Los procesos de conversión para diferentes desechos son dispuestos en un orden tal, que permite una adición mínima de energía externa y de materia prima, buscando lograr una máxima eficiencia en el proceso global. (Caicedo, 2001).

Una tecnología sostenible implica algo más que producir bienes sin contaminar ni destruir el medio ambiente. Significa satisfacer las necesidades de la gente de tal manera que no supere la capacidad de recuperación del planeta y de los ecosistemas locales. El objetivo consiste en limitar el uso de los recursos naturales del mundo dentro de los parámetros establecidos por la capacidad de recuperación de la tierra. (Mulder, 2007). Un sistema descentralizado de manejo de las aguas residuales puede definirse como la recolección, tratamiento, y vertimiento o reutilización de las aguas residuales provenientes de hogares, industrias o comunidades existentes cerca del punto de generación de residuos. Dado que una red completa de alcantarillado no es posible para muchos habitantes, es claro que el manejo descentralizado de aguas residuales es de gran importancia para el manejo futuro del ambiente. Por tanto, el concepto de manejo descentralizado de aguas residuales merece el mismo grado de atención que hasta ahora estaba reservado para los sistemas convencionales de manejo centralizado de aguas residuales. (Tchobanoglous, 2000). Actualmente hay un creciente interés por el desarrollo de nuevas tecnologías sostenibles, para el tratamiento de aguas residuales, que permiten obtener un efluente, a bajo costo con recuperación y uso de subproductos. (Glas).

En la Estación de Investigación y Transferencia de Tecnología de Aguas Residuales ubicada en Ginebra- Valle del Cauca-Colombia, se realizó una investigación, con el objetivo de evaluar el potencial de un humedal de flujo subsuperficial con Papiro, construido a escala piloto, como alternativa de post-tratamiento de aguas residuales domésticas, en términos de la eficiencia de remoción de coliformes fecales. Dentro de las conclusiones de la investigación se destaca que los procesos físico-químicos y biológicos que ocurren al interior del humedal, involucrados en los mecanismos de remoción de nutrientes y patógenos, son altamente influenciados por el $\mathrm{pH}$, la densidad de las plantas, la carga hidráulica, el tiempo de retención hidráulica (TRH) y las condiciones aerobias y anaerobias del suelo; esto se ve reflejado en las eficiencias obtenidas durante la fase experimental. En general, el porcentaje de nitrógeno retenido en el humedal es muy bajo durante la primera y segunda etapa del estudio. (Otero, 2001).En una investigación realizada en la planta de tratamiento de aguas residuales domésticas de La Vorágine, vereda perteneciente al Corregimiento de Pance del municipio de Santiago de Cali - Colombia. Se estudió el 
Desempeño de un sistema integrado de tratamiento de agua residual doméstica compuesto por tanque séptico, filtro anaerobio de flujo ascendente y Filtro fitopedológico, para establecer relaciones entre las variables de diseño que se adapten a la realidad local y sirvan de base para encarar racionalmente proyectos futuros. El sistema consta de dos líneas de tratamiento independientes, lo que ha permitido desarrollar el trabajo de una de ellas, bajo tres condiciones reales de funcionamiento. Finalmente la investigación permitió visualizar un potencial para el sistema evaluado de manera de ofrecer soluciones al problema de tratamiento de aguas residuales domésticas en los contextos rurales y urbanos marginales en el que se deben seleccionar tecnologías eficientes, confiables y simples en su operación y mantenimiento. (Rivera, 1998).

La Corporación Autónoma Regional del Valle del Cauca CVC, buscando alternativas económicas, fáciles de operar y mantener, ha obtenido resultados satisfactorios con el sistema conformado por tanque séptico y filtro anaerobio, con remociones superiores al $80 \%$ de DBO y SS. En el sistema conformado por decantador - humedal de flujo superficial, ha tenido problemas que están siendo remediados y que han permitido ir conociendo esta tecnología que es promisoria en nuestro medio. (Osorio, 2003)

CIPAV (2007), propone el tratamiento biológico con organismos acuáticos que aporta no solo la limpieza de las aguas para riego y lavado sino que ofrece ventajas económicas como la generación de abono, combustible, productos agrícolas y forrajes, integrados al sistema para alimentación humana y animal. El sistema integrado de producción agrícola - pecuario y descontaminación se encuentra en un área de $400 \mathrm{~m} 2$. Sus principales componentes son cerdos biodigestor - canales con plantas acuáticas - estanques de peces y/o riego - cultivos; lo han denominado descontaminación productiva.

Un humedal es un sistema que consiste en un estanque o canal poco profundo, construido por el hombre, para el tratamiento de aguas residuales, en el que se siembran plantas acuáticas; estas plantas ayudan a purificar el agua mediante la absorción de los nutrientes, eliminando una cantidad significativa de contaminantes, mientras sus raíces proporcionan el hábitat para microorganismos. El tratamiento en el humedal ocurre cuando el flujo de agua atraviesa lentamente el tallo y la raíz de la vegetación emergente, la cual permite la degradación biológica de contaminantes y materia orgánica por microorganismos, generando también la sedimentación de los sólidos suspendidos. (Thurnhofer, 2007). La figura 1 muestra un corte de un humedal (biofiltro de flujo horizontal) con heliconias.

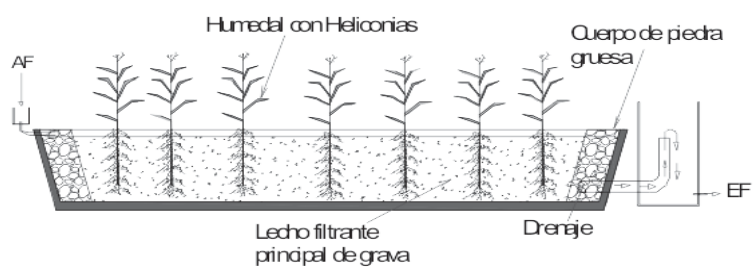

Figura. 1. Corte longitudinal de un Humedal de flujo horizontal.

En una evaluación realizada a un Biofiltro de Flujo Horizontal, construido en Masaya - Nicaragua, se encontraron remociones de $97 \%$ de DBO; $94 \%$ de DQO, $97 \%$ de SS, $34 \%$ de N, $26 \%$ de P, y 99\% de CF, considerando altas las remociones de DBO DQO y SS, aceptables las de N y P, y baja la de CF (Thurnhofer, 2007).

Una alberca biológica es un sistema que consiste en un tanque pequeño para el tratamiento de aguas residuales, donde se siembran plantas flotantes las cuales realizan el tratamiento. Este tanque está dividido en dos compartimientos donde se siembra Jacinto de agua (buchón de agua), las plantas funcionan como medio de filtración y adsorción de sólidos, poseen un sistema radicular sobre el cual las bacterias crecen las cuales ayudan a realizar la limpieza a la planta; el sistema esta complementado con un filtro compuesto de piedra, grava y arena, en el cual se culmina el tratamiento del efluente proveniente de las albercas con buchón. (Medina, 2007). La figura 2 muestra el corte longitudinal de una alberca biológica sembrada con Jacinto de agua. 


\section{Buchon de Agua}

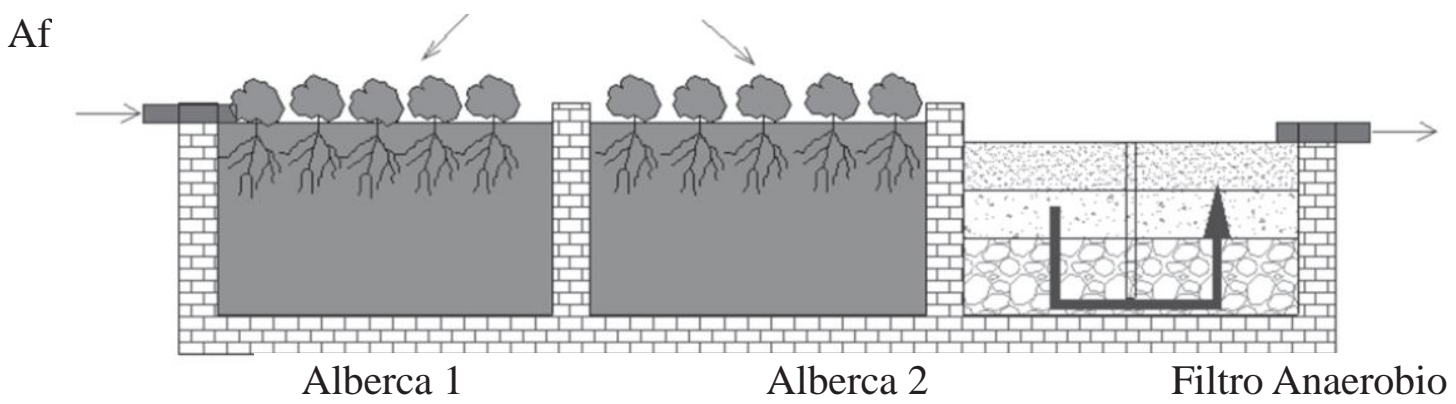

Figura. 2. Corte longitudinal de una Alberca Biológica.

En una Alberca Biológica construida para el tratamiento de las aguas residuales domésticas en la finca la Choza de la vereda el Porvenir del municipio de Pitalito Huila, se encontraron remociones de $28 \%$ de DBO, $47 \%$ de SS, $39 \%$ de grasas y aceites (G y A) y $90 \%$ de P, muy inferiores a las teóricas esperadas a excepción del P (Pinzón y Vélez, 2008). Así mismo, para una Alberca Biológica, construida en la Institución Educativa El Tejar del municipio de Timaná Huila, para el tratamiento de las aguas residuales de una porqueriza, se presenta como remociones teóricas, $83 \%$ de DBO, 77 de SS, $68 \%$ de N, 67\% de P y 99,999\% de CF. (Medina, 2007)

Un tanque séptico de acción múltiple es un sistema de tratamiento de aguas residuales, en el cual el tanque séptico se divide en dos compartimentos, complementados con un filtro anaerobio de flujo ascendente. La primera unidad está compuesta por un compartimiento de sedimentación donde las partículas pesadas van al fondo por gravedad y las livianas se dirigen hacia la superficie, conformando una capa delgada de espumas y natas. La segunda es un digestor, donde se depositan los sólidos sedimentados de la sección superior y se inicia su correspondiente biodegradación mediante un proceso anaerobio. El tratamiento secundario se da en el filtro anaerobio, donde el efluente forma una película biológicamente activa en los espacios que dejan los agregados, degradando la materia orgánica restante. (Valencia y Olaya, 1998). La figura 3 muestra un corte longitudinal de un TSAM.

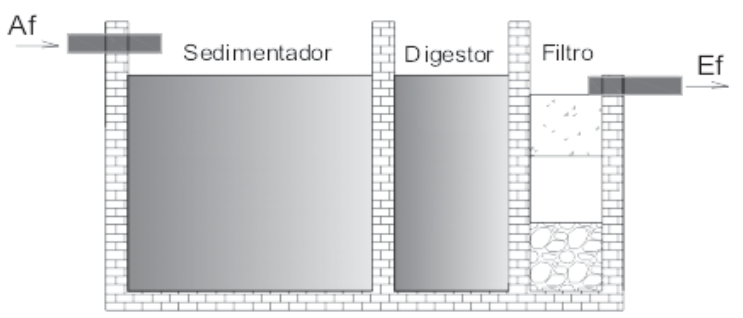

Figura. 3. Corte longitudinal de un TSAM

La eficiencia de remoción de DBO en los tanques sépticos depende de la temperatura, se pueden lograr remociones del orden del $70 \%$ de DBO y SS y $90 \%$ de CF. En el TSAM, las remociones pueden subir a $85 \%$ de DBO, $90 \%$ de SS y $99 \%$ de CF (Valencia y Olaya, 1998)

\section{Metodología}

\subsection{Localización}

Las propuestas están dirigidas a la zona rural del departamento del Huila y específicamente a las de minifundio, 
Para explotaciones agropecuarias menores a 10 hectáreas y de economía campesina, localizadas principalmente en zonas de ladera.

\subsection{Métodos}

\subsubsection{Trabajo de campo}

Se realizaron visitas a sistemas de tratamiento de aguas residuales construidos en zonas de minifundio del departamento del Huila, en los municipios de Neiva, Rivera, Pitalito y Palestina, donde se tomaron datos e información sobre las unidades de los sistemas, población, caudales, reuso y producción con efluentes.

\subsubsection{Trabajo de oficina}

Se realizó una revisión bibliográfica, referente al tratamiento de aguas residuales domesticas a pequeña escala, así como conceptos de aguas residuales, sistemas de tratamiento, y normas y parámetros de diseño; para elaborar un concepto sobre un sistema descentralizado integrado y sostenible para el tratamiento de aguas residuales domésticas. Con base al concepto elaborado, se diseñaron tres prototipos de sistemas descentralizados integrados y sostenibles para el tratamiento de aguas residuales domésticas. A cada propuesta se le realizaron los diseños conceptual y físico de acuerdo a lo establecido en el RAS 2000 y a normas y parámetro de diseño reportados por la literatura.

\section{Resultados}

\subsection{Concepto de Sistema Descentralizado Integrado y Sostenible para el tratamiento de aguas residuales domesticas}

Un sistema descentralizado integrado y sostenible para el tratamiento de aguas residuales domesticas en el sector rural es una solución individual para las aguas residuales de cada vivienda, donde se integra el tratamiento de las aguas residuales, con el reuso de los subproductos del tratamiento y los productos retornan a la vivienda beneficiando a sus pobladores. Al ser el sistema cíclico no genera residuos para disponer al ambiente, por lo tanto no se produce contaminación. Estos sistemas de tratamiento deben diseñarse de tal manera que sean amigables con el ambiente y los contaminantes no sean vertidos a las fuentes, convirtiéndose en materia prima para la producción; además deben ser de bajos costos y de fácil construcción operación y mantenimiento. Un sistema descentralizado integrado y sostenible se representa en la figura 4.
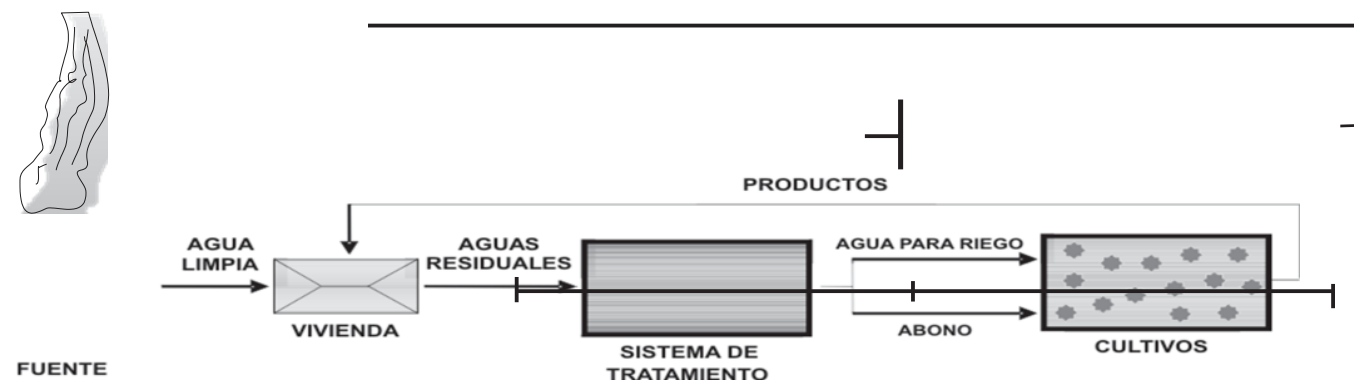

Figura. 4. Esquema general de un Sistema Descentralizado Integrado y Sostenible.

Generalmente para conducir las aguas residuales se utilizan los alcantarillados, que se caracterizan por ser de alto costo y más aún en el sector rural, debido a los grandes movimientos de tierra, instalación de tuberías y distancia entre las viviendas; lo que se agrava con la cultura de la gente, es costumbre disponer todo tipo de residuos a los alcantarillados. Con los sistemas descentralizados se minimiza este problema, no se construyen alcantarillados, al conducir las aguas residuales directamente al sistema de tratamiento.

Además en las zonas de minifundio y ladera, por razones de tipo topográfico y económico, no siempre es posible la construcción de grandes sistemas de alcantarillados y de tratamiento centralizados de aguas residuales; requiriéndose soluciones eficientes a las condiciones locales, lo que implica emplear sistemas descentralizados y no convencionales, que tengan en cuenta la cultura de la gente y que se centre en el uso de los residuos para la producción y el bienestar de los pobladores. 
Un sistema de tratamiento de aguas residuales es integrado cuando asocia un conjunto de partes (Tratamiento

- Reuso - Producción) enlazadas entre si y que llevan a un mismo fin, el manejo adecuado de las aguas residuales, minimizando su efecto contaminante. Se considera integrado un sistema cuando se contempla el Tratamiento de los afluentes, el Reuso de los subproductos del tratamiento en la Producción agropecuaria y los productos de esta son utilizados nuevamente por los pobladores para su beneficio directo o para su comercialización. Cambiando la idea común de que los residuos son un problema convirtiéndolos en un recurso.

Por ser un sistema sostenible, tiene la capacidad de tratar naturalmente las aguas residuales, sin requerimientos adicionales de energía. Desde el punto de vista económico los sistemas serán sostenibles si los costos de construcción, operación y mantenimiento son bajos, las unidades pueden ser construidas y operadas por las mismas comunidades y los subproductos del sistema generan recursos que permiten recuperar la inversión. Además contribuye a la sostenibilidad ambiental, al garantizar la calidad del recurso hídrico para futuras generaciones al no arrojar efluentes a las fuentes. Los sistemas de tratamiento de aguas residuales son sostenibles socialmente si las comunidades los aceptan y se apropian de ellos, convirtiéndose en sistemas sostenibles en el tiempo.

Los sistemas descentralizados integrados y sostenibles están conformados por una o varias unidades de sistemas no convencionales, siendo los más comunes en nuestro medio: los tanques sépticos, los humedales, los biodigestores, las albercas biológicas, los canales con plantas acuáticas y los filtros anaerobios.

\subsection{Prototipos de sistemas descentralizados integrados y sostenibles}

3.2.1. Sistema con humedales Contempla para el tratamiento preliminar una trampa de grasas para las aguas grises (AG), para el tratamiento primario un sedimentador y para el secundario un humedal sembrado con heliconias. El efluente (Ef) del humedal es usado para riego de un cultivo y la producción del humedal se destina su comercialización. La figura 5 muestra un esquema del sistema.

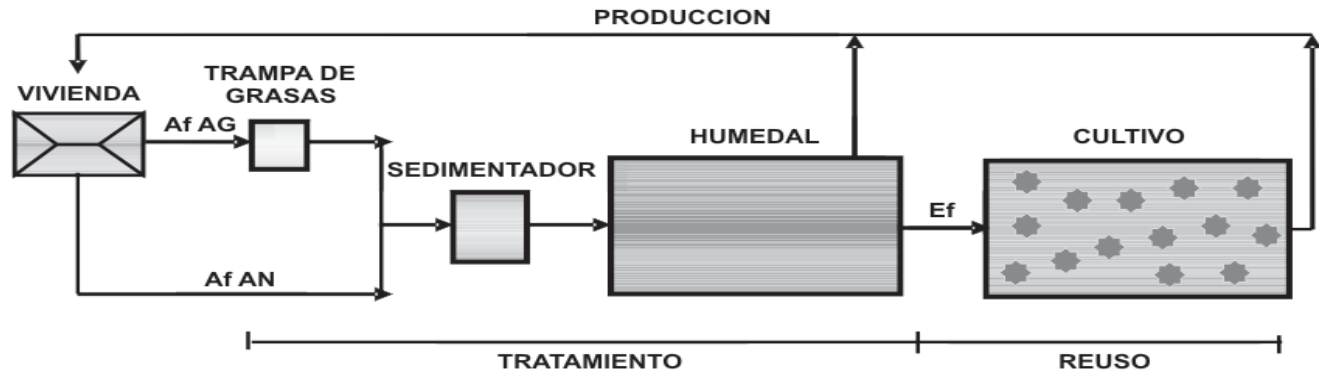

Figura. 5. Esquema del Sistema con Humedal

Las eficiencias teóricas de remoción de contaminantes del sistema, pueden considerarse altas, del $83 \%$ en DBO, $92 \%$ en SS y G y A, el $60 \%$ en N, $46 \%$ en P y $99 \%$ en CF.

\subsubsection{Sistema con Alberca Biológica}

Contempla para el tratamiento preliminar una trampa de grasas para las aguas grises, en el tratamiento primario un sedimentador y en el secundario una Alberca Biológica, compuesta por dos tanques sembrados con plantas acuáticas: Jacinto de agua Eichhornia Crassipes, y un filtro anaerobio, Los Jacintos cosechados serán compostados para ser utilizado en un cultivo y el efluente del filtro se utilizará para riego. La producción retorna a la vivienda para ser comercializada. La figura 6 muestra un esquema del sistema. 


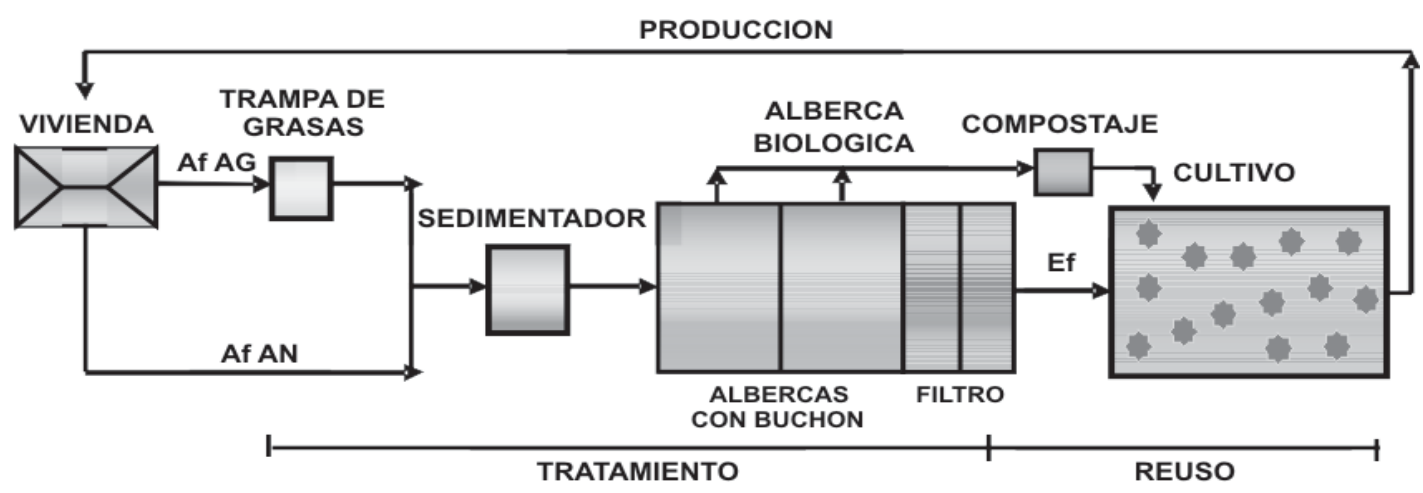

Figura.6. Esquema del Sistema con Alberca Biológica.

Las Eficiencias teóricas de remoción de contaminantes del sistema, pueden considerarse altas, 95\% en DBO, $98 \%$ en SS, $92 \%$ en G y A, $85 \%$ en N, 54\% en P y $99 \%$ en CF.

\subsubsection{Sistema con Tanque Séptico de Acción Múltiple (TSAM).}

Contempla para el tratamiento preliminar una trampa de grasas, y un Tanque Séptico de Acción Múltiple, conformado por un sedimentador como tratamiento primario y un digestor y un filtro anaerobio de flujo ascendente como tratamiento secundario. Los lodos extraídos del sedimentador serán compostados para ser utilizados como abono en un cultivo; y el efluente como agua para riego. La figura 7 muestra un esquema del sistema.

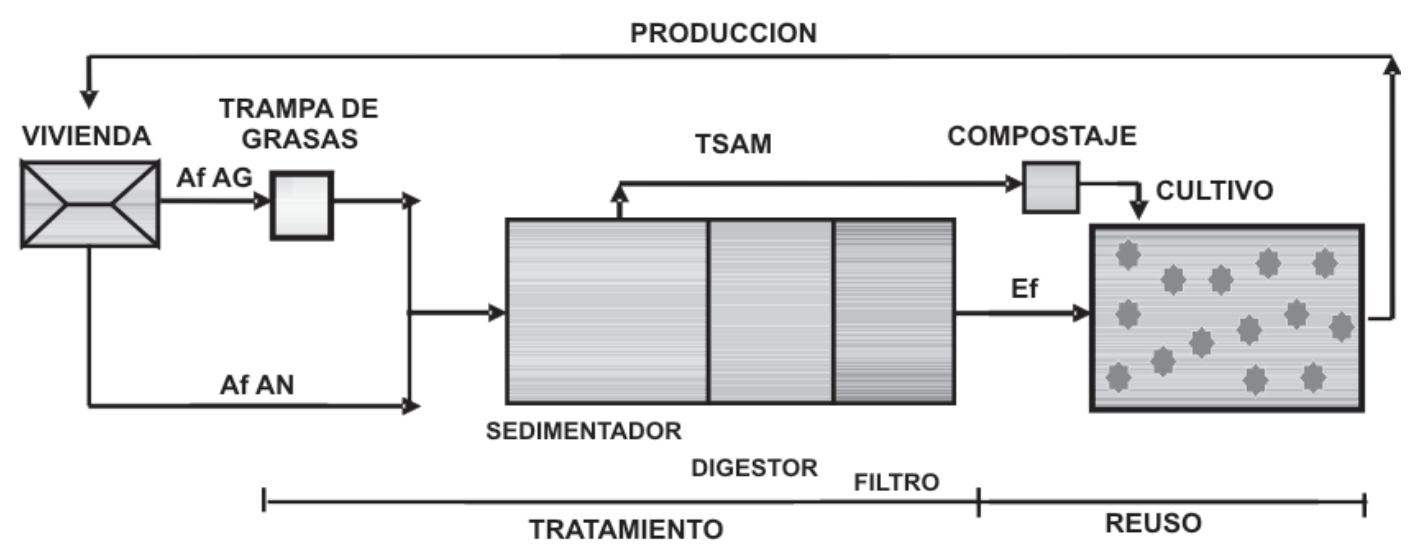

Figura. 7. Esquema del Sistema on Tanque Séptico de Acción Múltiple.

Las Eficiencias teóricas de remoción de contaminantes del sistema, pueden considerarse altas, $80 \%$ en DBO, $94 \%$ en SS, $92 \%$ en G y A, $62 \%$ en N, $58 \%$ en P y $99 \%$ en CF

\section{Conclusiones}

Los sistemas descentralizados integrados y sostenibles, son sistemas de bajo costo, de fácil operación y mantenimiento, que pueden ser construidos y operados por los mismos habitantes de la zona rural, convirtiéndose en una alternativa para el manejo de las aguas residuales en las zonas de minifundio del departamento del Huila. 
Los sistemas descentralizados integrados y sostenibles para el tratamiento de aguas residuales domésticas, reducen significativamente la contaminación de ríos y quebradas. Además minimizan los riesgos sobre la salud de los habitantes del sector rural, mejorando su calidad de vida y el entorno.

Los sistemas descentralizados integrados y sostenibles son concebidos como de fácil construcción, operación y mantenimiento; sin embargo para que cumplan los objetivos para los que se construyen, se requiere realizar las actividades de operación y mantenimiento necesarias que aseguren mantener su eficiencia.

Los prototipos de unidades propuestos como tratamiento secundario en este proyecto: Humedales, Alberca Biológica y TSAM, pueden ser construidos en el sector rural, sustentados en sus eficiencias, bajos costos y fácil construcción, operación y mantenimiento. En caso de requerir aumentar eficiencias se pueden complementar con otras unidades.

El tema de los sistemas descentralizados integrados y sostenibles para el tratamiento de aguas residuales en el sector rural, merece ser objeto de estudio e investigación, por su alto impacto en el medio ambiente y la calidad de vida de la gente.

\section{Referencias Bibliográficas}

1. Caicedo, J. R., 2001. Lagunas de Lenteja de Agua en Combinación con Reactores Anaeróbicos y Estanques Piscícolas: una Alternativa para el Tratamiento Sostenible de Aguas Residuales. Curso Internacional de Sistemas Integrados Sostenibles para el Tratamiento de Aguas Residuales. Universidad del Valle. Cali, Junio.

2. CIPAV, 2007. Descontaminación productiva del agua. Grupo Acuacultura. Cali.

3. Glas C., C., Evaluación de Factores Físicos, Químicos y Biológicos en el Desempeño de

Reactores Duckweed, con y sin Pretratamiento Anaerobio en el Tratamiento de Aguas

Residuales Domésticas.

4. Medina, P., A., 2007. Manejo de los Residuos de la Explotación Porcina en la Institución Educativa El Tejar Municipio de Timaná Huila. Diseño de una alberca biológica. Tesis. Universidad Surcolombiana. Neiva, p $44-47$

5. Mulder, K, 2007. Desarrollo sostenible para ingenieros.

6. Osorio, M., P., 2003. Sistemas de Tratamiento de Aguas Residuales Domésticas empleadas por la CVC en el Sector Rural del Departamento del Valle del Caica.

7. Otero, L., A., Marín, M., F., 2001. Evaluación de la Eficiencia de Remoción de Nutrientes y Coliformes Fecales de un Humedal de Flujo Subsuperficial con Papiro, en el PostTratamiento de Aguas Residuales Domésticas. CINARA, Universidad del Valle, Cali.

8. Pinzón, H, P, Vélez, Ch, E, 2008, Evaluación Preliminar de una Alberca Biológica como Sistema de Tratamiento de Aguas residuales Domésticas. Tesis. Universidad Surcolombiana. Neiva, p 43 - 44

9. Rivera, G., C., 1998. Evaluación del Tratamiento de Agua Residual Doméstica en un Sistema Integrado un Caso con Tanque Séptico, Filtro Anaerobio y Filtro Fitopedológico. CINARA, Universidad del Valle, Cali.

10. Tchobanoglous, G., Crites, R., 2000. Sistemas de Manejo de Aguas Residuales para Núcleos Pequeños y Descentralizados. Tomos 1, 2 y 3. Editorial Mc. Graw Hill, Bogotá.

11. Thurnhofer, M., Gauss, M., Cáceres, V., Fong. N., 2007. Biofiltro: Una Opción Sostenible para el Tratamiento de Aguas Residuales en Pequeñas Localidades. Programa de Agua y Saneamiento, América Latina y el Caribe (WSP-LAC). Honduras.

12. Valencia G, E., Olaya, M., M., 1997. Generalidades sobre Saneamiento Rural. Universidad Surcolombiana, Neiva, p $80-82$. 